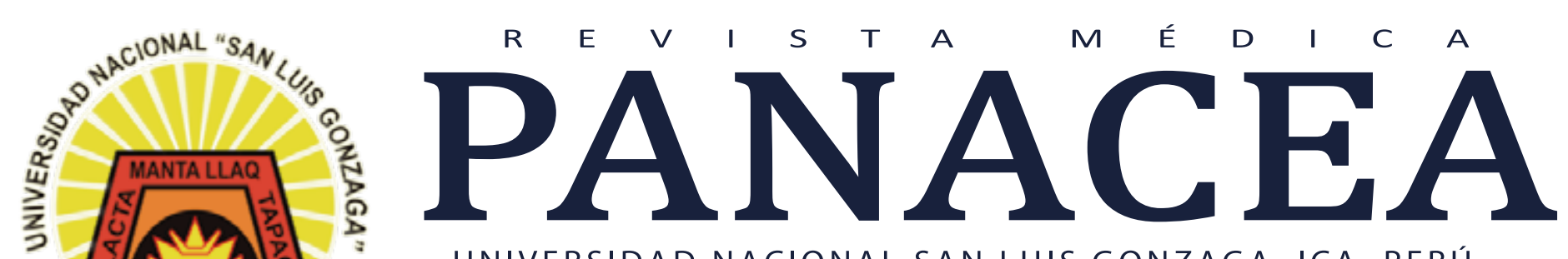

UNIVERSIDAD NACIONAL SAN LUIS GONZAGA. ICA, PERÚ

ICA HATUN YACHAY HUASI

FACULTAD DE MEDICINA HUMANA "DANIEL ALCIDES CARRIÓN"

p-ISSN 2223-2893

e-ISSN 2225-6989

Volumen 9 Número 2 PUBLICACION CUATRIMESTRAL Mayo - Agosto 2020

DOI: https://doi.org/10.35563/rmp.v9i2.322

\title{
EDITORIAL:
}

\section{EL PERSONAL DE SALUD EN LA PANDEMIA POR COVID-19A}

\author{
HEALTH STAFF IN THE COVID-19 PANDEMIC
}

\section{AUTORES:}

Jorge Ybaseta Medina

Bladimir Becerra Canales

INDEXADA EN:

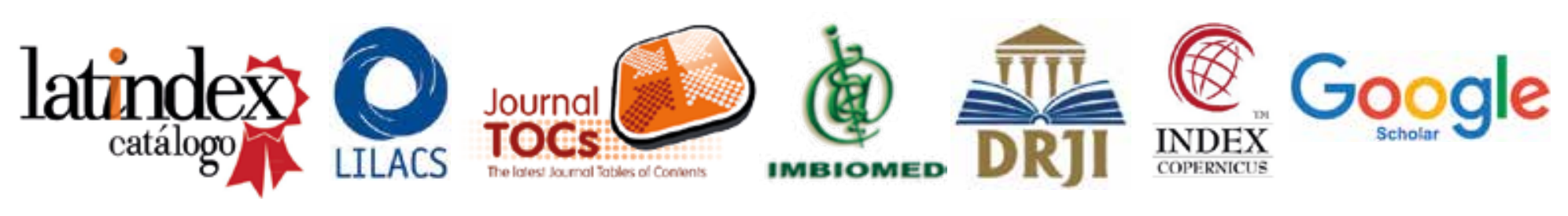

revistas.unica.edu.pe 


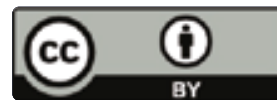

\title{
EL PERSONAL DE SALUD EN LA PANDEMIA POR COVID-19
}

\author{
HEALTH STAFF IN THE COVID-19 PANDEMIC
}

\author{
Jorge Ybaseta-Medina ${ }^{1, a}$, Bladimir Becerra-Canales ${ }^{1, b}$. \\ 1. Revista Médica Panacea. \\ a. Director. https://orcid.org/0000-0003-1224-1357 \\ b. Editor Asociado. https://orcid.org/0000-0002-2234-2189
}

\section{DOI: https://doi.org/10.35563/rmp.v9i2.322}

Actualmente se vive una crisis sanitaria de alcance global, catalogada por la Organización Mundial de la Salud (OMS) como pandemia (1). Se reportan al 26 de julio del 2020: 646996 víctimas mortales y sobrepasa los dieciséis millones de casos confirmados en el mundo (2) En Sudamérica el Perú, después de Brasil, ocupa el segundo lugar con 379,884 casos y 18,030 defunciones (2).

Por las características propias de esta pandemia, el personal de salud se enfrenta a estresores laborales intensos, tales como largas jornadas de trabajo, sobrecarga laboral, instrucciones y medidas de seguridad estrictas, necesidad permanente de concentración y vigilancia, escasez de equipamientos de protección entre otros $(3,4)$.

Se incluye además el colapso de un sistema sanitario precario, una mayor duración de la cuarentena, frustración, aburrimiento, pérdidas financieras (5) información insuficiente y poco claras para poder tomar acciones adecuadas $(6,7)$ percepción de falta de transparencia de los funcionarios públicos y del gobierno sobre la gestión de los recursos públicos (7).

Esta situación, pone en riesgo la salud física y mental del personal sanitario, dando lugar a contagios por inadecuadas condiciones de bioseguridad e infraestructura sanitaria, complicaciones a su propia salud y muerte. La afectación psicológica se manifiesta con síntomas de ansiedad, depresión o trastornos por estrés postraumático (8) o traumatización vicaria derivada de la compasión hacia los pacientes que están atendiendo (9).

Por otro lado se ha documentado en un estudio en 63 países, que el estado de ánimo de la población está cambiando en contra de los trabajadores de la salud, se han documentado agresiones, maltratos, daño físico, entre otras acciones (10).

En los últimos días los casos nuevos y defunciones se han incrementado rápidamente que ha complicado el manejo de los hospitales en el país. Sabemos que el primer nivel de atención o atención primaria es el pilar fundamental de un sistema de salud efectivo. La inhabilitación de este importante componente del sistema, limitándola solo a realizar referencias en etapas iniciales del brote, ha ocasionado el incremento de la presión asistencias a nivel hospitalario, complicaciones masivas de salud y colapso de nuestro sistema de salud precario. La lucha contra el COVID-19, necesariamente tiene que ser "puerta a puerta" y multidisciplinaria es decir con la participación de todo el equipo de salud, trabajando de manera coordinada desde el primer nivel de atención, la cual debe ser fortalecida en forma real y sostenida.

El impacto de la COVID-19 y sus implicancias, supone un reto importante para al personal sanitario, por la naturaleza propia de su trabajo, muchas veces en condiciones inseguras por el riesgo de contagio y psíquicamente exigentes. Se exhorta a las autoridades de turno de las instituciones involucradas (Gobierno Regional, Ministerio de Salud, Direcciones Regionales y otras dependencias administrativas del sector salud, Universidades, Colegios Profesionales, entre otros.), a tomar acciones efectivas respecto a esta problemática. 


\section{REFERENCIAS BIBLIOGRÁFICAS}

1. Sohrabi C, Alsafi Z, O'Neill $N$, Khan M, Kerwan $A$, Al-Jabir A, et al. World Health Organization declares global emergency: A review of the 2019 novel coronavirus (COVID-19). Int J Surg. 2020 [acceso: 26/07/2020];76:71-6. Disponible en: http://www.sciencedirect.com/science/article/pii/S 1743919120301977

2. COVID-19 Map. Maryland: Johns Hopkins Coronavirus Resource Center; 2020 [acceso: 26/07/2020]. Disponible en: https://coronavirus.jhu.edu/map.html

3. Vieta $E$, Pérez $V$, Arango C. Psychiatry in the aftermath of COVID-19. Rev Psiquiatr Salud Ment. 2020;20:1-4, http://dx.doi.org/10.1016/j.rpsm.2020.04.004.

4. Brooks SK, Webster RK, Smith LE, Woodland L, Wessely S, Greenberg N, et al. The psychological impact of quarantine and how to reduce it: Rapid review of the evidence. The Lancet. 2020;395:912-20.

http://dx.doi.org/10.1016/S0140-6736(20)30460-8.

5. Bedford J, Enria D, Giesecke J, Heymann DL, Ihekweazu C, Kobinger G, et al. COVID-19: towards controlling of a pandemic [Internet]. Vol. 395, The Lancet. Lancet Publishing Group; 2020 [acceso: 26/07/2020]; 1015-8.

6. Reynolds DL, Garay JR, Deamond SL, Moran MK, Gold W, Styra R. Understanding, compliance and psychological impact of the SARS quarantine experience. Epidemiol Infect [Internet]. 2008 [acceso: 26/07/2020];136(7):997-1007.

7. Braunack-Mayer A, Tooher R, Collins JE, Street JM, Marshall $\mathrm{H}$. Understanding the school community's response to school closures during the H1N1 2009 influenza pandemic [Internet]. Vol. 13, BMC Public Health. 2013 [acceso: 26/07/2020]. 344.

8. Bao Y, Sun Y, Meng S, Shi J, Lu L. 2019-nCoV epidemic: Address mental health care to empower society. The Lancet. 2020;395(10224):e37-8. http://dx.doi.org/10.1016/S0140-6736(20)30309-3.

9. Li Z, Ge J, Yang M, Feng J, Qiao M, Jiang R, et al. Vicarious traumatization in the general public, members, and non-members of medical teams aiding in COVID-19 control. Brain Behav Immun. 2020; S0889-1591(20)30309-3. https://doi.org/10.1016/j.bbi.2020.03.007.

10. Global: El personal sanitario, silenciado, expuesto y atacado. [acceso: 26/07/2020]. Disponible en: https://www.amnesty.org/es/latest/news/2020/07/ health-workers-rights-covid-report/

\section{Cómo citar}

Ybaseta-Medina J, Becerra-Canales B. El personal de salud en la pandemia por COVI-19. Rev méd panacea.2020;(2):72-73.

DOI: https://doi.org/10.35563/rmp.v9i2.322 